\title{
INCLUSION THEOREMS FOR CONGRUENCE SUBGROUPS
}

BY

\author{
M. NEWMAN AND I. REINER(1)
}

1. Introduction. We shall use the following notation throughout: $A^{(r)}$ denotes an $r \times r$ matrix $A ; I^{(r)}$ denotes the $r$-rowed identity matrix; 0 will be used for a zero matrix of appropriate size. Congruence of matrices will be interpreted as elementwise congruence. We write $a \mid b$ to indicate that $a$ divides $b$. Lower case italic letters will always denote integers.

Let $G_{t}$ be the proper unimodular group consisting of all $t \times t$ matrices with integral elements and determinant +1 . For a fixed partition: $t=r+s$ of $t$ in to two positive integers $r$ and $s$, and for a fixed positive integer $n$, define the subgroup

$$
G_{r, s}(n)=\left\{\left[\begin{array}{ll}
A^{(r)} & B \\
C & D^{(s)}
\end{array}\right] \in G_{t}: \quad C \equiv 0(\bmod n)\right\} .
$$

We shall prove:

THEOREM 1. Let $m, n$ be positive integers, and let $H$ be a group such that

$$
G_{r, s}(m n) \subset H \subset G_{r, s}(n) .
$$

Then there exists a divisor $d$ of $m$ such that

$$
H=G_{r, s}(d n) .
$$

Special cases of this have been proved in [1] and [3].

In the case where $t=2 r$, define

$$
G_{r}(m, n)=\left\{\left[\begin{array}{ll}
A^{(r)} & B \\
C & D^{(r)}
\end{array}\right] \in G_{2 r}: \begin{array}{l}
B \equiv 0(\bmod m) \\
C \equiv 0(\bmod n)
\end{array}\right\} .
$$

Then we shall show:

Theorem 2. Let $H$ be a group satisfying

$$
G_{r}(m, n) \subset H \subset G_{2 r} .
$$

If $(m, n)=1$, then there exist integers $m_{1}, n_{1}$ with $m_{1}\left|m, n_{1}\right| n$, and

$$
H=G_{r}\left(m_{1}, n_{1}\right) \text {. }
$$

Presented to the Society, October 26, 1957; received by the editors October 14, 1957.

(1) The work of the first author was supported (in part) by the Office of Naval Research, and that of the second author was supported (in part) by a contract with the National Science Foundation. 
A special case of this (with $r=1$ ) was proved in [2], where it was also shown that the hypothesis $(m, n)=1$ could not be dropped.

To generalize further, let $\mathfrak{n}=\left(n_{1}, \cdots, n_{t-1}\right)$, and define

$$
G_{t}(\mathfrak{n})=G_{1, t-1}\left(n_{1}\right) \cap G_{2, t-2}\left(n_{2}\right) \cap \cdots \cap G_{t-1,1}\left(n_{t-1}\right) .
$$

Thus an element $M \in G_{t}$ lies in $G_{t}(\mathfrak{n})$ if and only if for every partition $t=r+s(1 \leqq r \leqq t-1)$ we have

$$
M=\left[\begin{array}{ll}
A^{(r)} & B \\
C & D^{(s)}
\end{array}\right], \quad C \equiv 0\left(\bmod n_{r}\right) .
$$

We shall prove:

Theorem 3. Let $\left(m_{i} n_{i}, m_{j} n_{j}\right)=1$ for $1 \leqq i, j \leqq t-1, i \neq j$. Let $H$ be a group such that

$$
G_{t}(\mathfrak{m n}) \sqsubset H \subset G_{t}(\mathfrak{n}),
$$

where $m \mathfrak{n}$ denotes $\left(m_{1} n_{1}, \cdots, m_{t-1} n_{t-1}\right)$. Then there exists a vector

$$
\mathfrak{D}=\left(d_{1}, \cdots, d_{t-1}\right) \text {, }
$$

with $d_{1}\left|m_{1}, \cdots, d_{t-1}\right| m_{t-1}$, such that

$$
H=G_{t}(\mathfrak{d n}) .
$$

Finally, we shall prove analogues of Theorems 1 and 2 for the symplectic modular group $\Gamma_{t}$ of order $t$, which consists of all integral matrices

$$
\left[\begin{array}{ll}
A^{(t)} & B \\
C & D^{(t)}
\end{array}\right]
$$

satisfying

$$
A B^{\prime}=B^{\prime} A, \quad C D^{\prime}=D^{\prime} C, \quad A D^{\prime}-D C^{\prime}=I .
$$

2. We begin the proof of Theorem 1 with two lemmas.

Lemma 1. Let $t=r+s$, and let $n$ be a fixed positive integer. For each

$$
M=\left[\begin{array}{ll}
A^{(r)} & B \\
C & D^{(s)}
\end{array}\right] \in G_{t}
$$

there cxists an integral $r \times$ s matrix $X$ such that $(|A+X C|, n)=1$.

Proof. It is sufficient to show that for every prime $p$ there exists an integral matrix $X_{p}$ such that $p \nmid\left|A+X_{p} C\right|$. For we may then find an integral matrix $X$ satisfying $X \equiv X_{p}(\bmod p)$ for each $p \mid n$. Since $|A+X C|$ $\equiv\left|A+X_{p} C\right|(\bmod p)$, it then follows that $(|A+X C|, n)=1$.

Now let $p$ be a fixed prime, and let $\alpha_{1}, \cdots, \alpha_{r}$ denote the rows of $A$, and 
$\gamma_{1}, \cdots, \gamma_{s}$ those of $C$. Since the rows of $X_{p} C$ are linear combinations of those of $C$, we need only show that there exist linear combinations

$$
\beta_{i}=\sum_{j=1}^{s} x_{i j} \gamma_{j} \quad\left(1 \leqq i \leqq r, x_{i j} \text { integers }\right)
$$

such that $p \nmid \operatorname{det}\left(\alpha_{i}+\beta_{i}\right)$. Thus, we seek integers $x_{i j}$ for which the vectors $\alpha_{i}+\beta_{i}(1 \leqq i \leqq r)$ are linearly independent modulo $p$.

Since $M$ is unimodular, the set $\left\{\alpha_{1}, \cdots, \alpha_{r}, \gamma_{1}, \ldots, \gamma_{s}\right\}$ contains exactly $r$ linearly independent vectors modulo $p$. Suppose that $r^{\prime}$ of the $\alpha$ 's are linearly independent modulo $p\left(r^{\prime} \leqq r\right)$; for simplicity of notation, suppose that these are $\alpha_{1}, \cdots, \alpha_{r^{\prime}}$. Then each $\alpha_{k}\left(r^{\prime}<k \leqq r\right)$ is a linear combination modulo $p$ of $\alpha_{1}, \cdots, \alpha_{r^{\prime}}$. Further, there exist $r-r^{\prime}$ vectors $\gamma_{1}^{*}, \cdots, \gamma_{r-r^{\prime}}^{*}$ among $\gamma_{1}, \cdots, \gamma_{s}$ such that the set $\left\{\alpha_{1}, \cdots, \alpha_{r^{\prime}}, \gamma_{1}^{*}, \cdots, \gamma_{r-r^{\prime}}^{*}\right\}$ is linearly independent modulo $p$. Then we need only choose $\beta_{1}=\cdots=\beta_{r^{\prime}}=0, \beta_{r^{\prime}+1}$ $=\gamma_{1}^{*}, \cdots, \beta_{r}=\gamma_{r-r^{\prime}}^{*}$ to achieve the desired result.

Lemma 2. Let $M \in G_{r, s}(n)$, and let $m$ be a fixed positive integer. Then there exists an integral $r \times s$ matrix $X$ and an integral $s \times r$ matrix $Y$ such that

$$
W(n Y) S(X) M \in G_{r, s}(m n),
$$

where

$$
W(n Y)=\left[\begin{array}{ll}
I^{(r)} & 0 \\
n Y & I^{(s)}
\end{array}\right], \quad S(X)=\left[\begin{array}{ll}
I^{(r)} & X \\
0 & I^{(s)}
\end{array}\right] .
$$

The entries of $X$ and $Y$ are integers determined only modulo $m$. Therefore the set of products $W(n Y) S(X)$, as the entries of $X$ and $Y$ range over all residues modulo $m$, contains a full set of left coset representatives of $G_{r, s}(n)$ modulo $G_{r, s}(m n)$. Consequently $G_{r, s}(m n)$ is of finite index in $G_{r, s}(n)$.

Proof. Set

$$
M=\left[\begin{array}{ll}
A^{(r)} & B \\
n C & D^{(s)}
\end{array}\right] \in G_{r, s}(n) .
$$

By Lemma 1, we can determine $X$ modulo $m$ such that $(|A+n X C|, m)=1$. Set $A_{0}=A+n X C$. Then

$$
S(X) M=\left[\begin{array}{ll}
A_{0} & * \\
n C & *
\end{array}\right]
$$

and

$$
W(n Y) S(X) M=\left[\begin{array}{cc}
* & * \\
n\left(Y A_{0}+C\right) & *
\end{array}\right] .
$$


In order for (10) to hold, we need only show that $Y$ modulo $m$ can be determined so that $Y A_{0}+C \equiv 0(\bmod m)$.

Now $\left(\left|A_{0}\right|, m\right)=1$, so that we may find an integer $a$ with $a\left|A_{0}\right| \equiv 1$ $(\bmod m)$. Letting $A_{0}^{\text {adj }}$ denote the adjoint of $A_{0}$, we set

$$
Y \equiv-a C A_{0}^{\text {adj }}(\bmod m) \text {. }
$$

Using $A_{0}^{\text {adj }} A_{0}=\left|A_{0}\right| I$, we obtain

$$
Y A_{0} \equiv-C(\bmod m)
$$

as desired.

The remainder of the lemma follows at once from (10).

We now proceed with the proof of Theorem 1 . Let $H$ be a group such that

$$
G_{r, s}(m n) \subset H \subset G_{r, s}(n) .
$$

Using the argument in [1], we find by induction on the total number of prime factors of $m$ that the conclusion of Theorem 1 is valid unless for every $d$ dividing $m, d \neq 1$, we have

$$
H \cap G_{r, s}(d n)=G_{r, s}(m n) .
$$

Suppose now that $H \neq G_{r, 8}(m n)$. The above then shows that there exists a matrix

$$
M=\left[\begin{array}{ll}
A^{(r)} & B \\
n C & D^{(s)}
\end{array}\right] \in H
$$

such that $C \not \equiv 0(\bmod d)$ for any divisor $d$ of $m, d \neq 1$. Choose $X, Y$ as in Lemma 2, and use the fact that $S(X) \in H$. Then we see that $W(n Y) \in H$, where $Y$ is chosen by use of (11). Hence also $Y \not \equiv 0(\bmod d)$ for any divisor $d$ of $m, d \neq 1$.

Call an $s \times r$ matrix $T$ permissible if $W(n T) \in H$. We have shown the existence of a permissible matrix $Y$ such that $Y \not \equiv 0(\bmod d)$ for any divisor $d$ of $m$, $d \neq 1$. We shall use this to deduce that every matrix is permissible. Since already $S(X) \in H$ for all $X$, it will then follow from Lemma 2 that $H=G_{r, s}(n)$, and the theorem will be proved.

Now we have

$$
W\left(n T_{1}\right) \cdot W\left(n T_{2}\right)=W\left(n\left(T_{1}+T_{2}\right)\right)
$$

and

$$
\left[\begin{array}{ll}
V^{-1} & 0 \\
0 & U
\end{array}\right] W(n T)\left[\begin{array}{ll}
V & 0 \\
0 & U^{-1}
\end{array}\right]=W(n U T V), U \in G_{s}, V \in G_{r} .
$$

Therefore if $T_{1}$ and $T_{2}$ are permissible, so is $T_{1}+T_{2}$. If $T$ is permissible, then 
so is $-T$; and if $U \in G_{s}, V \in G_{r}$, then $U T V$ is also permissible.

Starting with the permissible $Y$ above, set $Y_{1}=U Y V$, with $U \in G_{s}, V \in G_{r}$. Then $Y_{1}$ is also permissible, and with proper choice of $U$ and $V$, we may take $Y_{1}$ in Smith normal form:

$$
Y_{1}=\left[\begin{array}{llll}
h_{1} & & & \\
& h_{2} & \\
& & \ddots & \\
& & h_{\mu}
\end{array}\right], \quad \mu=\min (r, s),
$$

where $h_{1}\left|h_{2}\right| \cdots \mid h_{\mu}$. If $\left(h_{1}, m\right)>1$, then there is a prime $p \mid m$ such that $Y_{1} \equiv 0(\bmod p)$. Then also $Y \equiv 0(\bmod p)$, which is impossible. Hence $\left(h_{1}, m\right)$ $=1$. Let us choose $a$ so that $a h_{1} \equiv 1(\bmod m)$. Then $Y_{2}=a Y_{1}$ is also permissible. Since a permissible matrix remains permissible when multiples of $m$ are added to its entries, we therefore have the permissible matrix

$$
Y_{3}=\left[\begin{array}{llll}
1 & & & \\
& k_{2} & & \\
& & \ddots & \\
& & & k_{\mu}
\end{array}\right] .
$$

Hence also

$$
Y_{4}=\left[\begin{array}{ccccc}
0 & -k_{2} & & & \\
1 & 0 & & & \\
& & k_{3} & \cdot & \\
& & & \ddots & \\
& & & & k_{\mu}
\end{array}\right]
$$

and

$$
Y_{5}=Y_{3}-Y_{4}=\left[\begin{array}{rrrrr}
1 & k_{2} & & & \\
-1 & k_{2} & & & \\
& & 0 & \\
& & & \ddots & \\
& & & & 0
\end{array}\right]
$$

are permissible. In $Y_{5}$ add the second row to the first row, and then subtract the matrix so obtained from $Y_{5}$, obtaining the permissible matrix which has 1 in the $(1,1)$ place, $-k_{2}$ in the $(1,2)$ place, and 0 elsewhere. In this matrix add $k_{2}$ times the first column to the second column, thereby obtaining the permissible matrix 


$$
Y_{6}=\left[\begin{array}{lllll}
1 & & & & \\
& 0 & & \\
& & \cdot & \\
& & & \cdot & \\
& & & 0
\end{array}\right] .
$$

Since also $U Y_{6} V$ is permissible for all $U \in G_{s}, V \in G_{r}$, we find that every matrix whose entries are all zeros except for a single 1 , must be permissible. Therefore all matrices are permissible, and Theorem 1 is proved.

3. We now prove Theorem 2. Let $H$ be a group satisfying

$$
G_{r}(m, n) \subset H \subset G_{2 r},
$$

where $G_{r}(m, n)$ is defined by (4), and where $(m, n)=1$. Choose integers $a, b$ satisfying $a m-b n=1$, and set

$$
K=\left[\begin{array}{ll}
a m I^{(r)} & I \\
b n I & I^{(r)}
\end{array}\right] \in G_{2 r} .
$$

Then as in [2] we find that $K^{-1} G_{r}(m, n) K=G_{r, r}(m n)$, and the remainder of the proof of Theorem 2 follows from Theorem 1 just as in [2].

Theorem 2 is false for $(m, n)>1$, as is shown in [2].

4. To prove Theorem 3 , we begin with several lemmas.

Lemma 3. Let $n_{1}, \cdots, n_{t-1}$ be pairwise coprime, and let $M \in G_{t}$. Then there exists an upper triangular matrix $S \in G_{t}$ such that for each $r(1 \leqq r \leqq t-1)$ we have

$$
M=\left[\begin{array}{ll}
A^{(r)} & B \\
C & D^{(t-r)}
\end{array}\right], \quad S \equiv\left[\begin{array}{ll}
I^{(r)} & X_{r} \\
0 & I^{(t-r)}
\end{array}\right]\left(\bmod n_{r}\right)
$$

and

$$
\left(\left|A^{(r)}+X_{r} C\right|, n_{r}\right)=1 \text {. }
$$

Proof. Let $M$ be fixed. For each $r$, write $M$ in the form (12). By Lemma 1, we may then choose $X_{r}$ such that (13) holds. We then use the Chinese remainder theorem to determine an upper triangular matrix $S$ satisfying

$$
S \equiv\left[\begin{array}{ll}
I^{(r)} & X_{r} \\
0 & I^{(t-r)}
\end{array}\right]\left(\bmod n_{r}\right), \quad 1 \leqq r \leqq t-1 .
$$

This completes the proof of the lemma.

Lemma 4 . Let $S$ be an integral $t \times t$ matrix such that $|S| \equiv 1(\bmod n)$. Then there exists a matrix $T \in G_{\iota}$ such that $T \equiv S(\bmod n)$.

Proof. (Although this lemma is known, references are hard to come by, and so we insert a proof.) 
Set $T=S+n Y$; we need only choose $Y$ so that $|S+n Y|=1$. Let $U, V \in G_{\boldsymbol{t}}$ be chosen so that $U S V=D$ is diagonal, and set $X=U Y V$. Then

$$
|S+n Y|=|D+n X| \text {, }
$$

so it suffices to show that we can find $X$ such that $|D+n X|=1$, where $D$ is diagonal and $|D| \equiv 1(\bmod n)$.

Let $D=\operatorname{diag}\left(d_{1}, \cdots, d_{t}\right)$, and set $|D|=1+n d$. Choose $X$ so that

$$
D+n X=\left[\begin{array}{cccccc}
d_{1}+n x & 0 & 0 & \cdots & 0 & n y \\
n & d_{2} & 0 & \cdots & 0 & 0 \\
0 & n & d_{3} & \cdots & 0 & 0 \\
. & . & . & \cdots & . \\
0 & 0 & 0 & \cdots & n & d_{t}
\end{array}\right] .
$$

Then

$$
|D+n X|=1+n\left(d+x d_{2} \cdots d_{t} \pm n^{t-1} y\right) .
$$

Since $\left(d_{2} \cdots d_{t}, n\right)=1$, we may choose integers $x, y$ such that

$$
d+x d_{2} \cdots d_{t} \pm n^{t-1} y=0,
$$

which completes the proof.

Lemma 5. Let $\mathfrak{m}=\left(m_{1}, \cdots, m_{t-1}\right), \mathfrak{n}=\left(n_{1}, \cdots, n_{t-1}\right)$, where $\left(m_{i}, n_{i}\right)=1$ for $1 \leqq i \leqq t-1,\left(m_{i} n_{i}, m_{j} n_{j}\right)=1$ for $1 \leqq i, j \leqq t-1, i \neq j$, and let $M \in G_{r}(\mathfrak{n})$. Then there is an upper triangular matrix $S \in G_{t}$ and a lower triangular matrix $W \in G_{t}$ such that $W S M \in G_{r}(\mathrm{mn})$. The entries of $W$ and $S$ are determined only modulo $m_{1} \cdots m_{t-1}$, and hence $G(\mathrm{mn})$ is of finite index in $G(\mathfrak{n})$.

Proof. This lemma follows readily from Lemma 3 in the same way that Lemma 2 follows from Lemma 1.

We now proceed with the proof of Theorem 3 . Let $\mathfrak{m}, \mathfrak{n}$ be chosen as in the above lemma, and let $H$ be a group such that

$$
G_{t}(\mathfrak{m n}) \subset H \subset G_{t}(\mathfrak{n}) .
$$

As in the proof of Theorem 1, by using induction on the total number of prime factors of $m_{1} m_{2} \cdots m_{t-1}$, we see that the theorem holds unless for every vector $\mathfrak{a}=\left(a_{1}, \cdots, a_{t-1}\right)$ such that $a_{1}\left|m_{1}, \cdots, a_{t-1}\right| m_{t-1}$, except

$$
\mathfrak{a}=(1, \cdots, 1) \text {, }
$$

we have

$$
H \cap G_{t}(\mathfrak{a n})=G_{t}(\mathfrak{n m}) .
$$

Suppose that $H \neq G_{t}(\mathfrak{m n})$; then $H$ must contain an element $M$ such that for each $r(1 \leqq r \leqq t-1)$ we have 


$$
M=\left[\begin{array}{ll}
A^{(r)} & B \\
n_{r} C & D^{(t-r)}
\end{array}\right]
$$

with $C \not \equiv 0\left(\bmod a_{r}\right)$ for each divisor $a_{r}$ of $m_{r}, a_{r} \neq 1$.

Now choose an upper triangular matrix $S$ and a lower triangular matrix $W$ as in Lemma 5 , such that $W S M \in G_{r}(m \mathfrak{m}) \subset H$. Since also $S \in H$, this shows that $W \in H$. Further, for each $r$ we have

$$
W \equiv\left[\begin{array}{ll}
I^{(r)} & 0 \\
n_{r} Y_{r} & I^{(t-r)}
\end{array}\right] \quad\left(\bmod m_{r}\right)
$$

where $Y_{r} \not \equiv 0\left(\bmod a_{r}\right)$ for any $a_{r}$ dividing $m_{r}, a_{r} \neq 1$.

Call a lower triangular matrix in $G_{t}$ permissible if it is an element of $H$. The above-constructed $W$ is permissible. If we can show that all lower triangular matrices in $G_{t}(\mathfrak{n})$ are permissible, then using Lemma 5 we will deduce that $H=G_{t}(\mathfrak{n})$, and Theorem 3 will be established.

Define the non-negative integer $k$ by $m_{1}=\cdots=m_{k-1}=1, m_{k}>1$. (If $m_{1}>1$, then choose $k=1$.) We shall show that also $m_{k+1}=\cdots=m_{t-1}=1$. For let $m_{0}=m_{k+1} \cdots m_{t-1}$; then $\left(m_{0}, m_{k}\right)=1$.

Now we remark that the matrix $Y_{r}$ was determined only modulo $m_{r}$, and hence since $\left(m_{r}, n_{r}\right)=1$, we could have chosen the permissible matrix $W$ so that instead of (15) we have (for each $r$ )

$$
W \equiv\left[\begin{array}{ll}
I^{(r)} & 0 \\
n_{r} Y_{r} & I^{(t-r)}
\end{array}\right] \quad\left(\bmod m_{r} n_{r}\right) .
$$

Then $W \in H$, so also $W^{m_{0}} \in H$. Now for each $r(1 \leqq r \leqq t-1)$ we have

$$
W^{m_{0}} \equiv\left[\begin{array}{ll}
I^{(r)} & 0 \\
n_{r} m_{0} Y_{r} & I^{(t-r)}
\end{array}\right] \quad\left(\bmod m_{r} n_{r}\right)
$$

whence

$$
W^{m_{0}} \in G_{t}\left(n_{1}, \cdots, n_{k}, m_{k+1} n_{k+1}, \cdots, m_{t-1} n_{t-1}\right) .
$$

Unless $\left(1, \cdots, 1, m_{k+1}, \cdots, m_{t-1}\right)=(1, \cdots, 1)$, we deduce from (15) that $W^{m_{0}} \in G_{t}(\mathrm{mn})$, which is impossible because $W^{m_{0}} \notin G_{k-1, t-k+1}\left(m_{k} n_{k}\right)$. We thus have shown that $\mathfrak{m}=\left(1, \cdots, 1, m_{k}, 1, \cdots, 1\right)$.

We are now supposing that

$$
G_{t}(\mathfrak{m n}) \subset H \subset G_{t}(\mathfrak{n}),
$$

where $\mathfrak{m}=\left(1, \cdots, 1, m_{k}, 1, \cdots, 1\right), m_{k}>1$, that (14) holds, and that $H \neq G_{t}(\mathfrak{m n})$. We have shown the existence of a lower triangular matrix $W \in H$ such that $(16)$ holds, with $Y_{k} \neq 0\left(\bmod a_{k}\right)$ for any $a_{k}$ dividing $m_{k}, a_{k} \neq 1$. We are trying to prove that every lower triangular matrix in $G_{t}(\mathfrak{n})$ is permissible (that is, lies in $H$ ), and consequently that $H=G_{t}(\mathfrak{n})$. 
Let $U \in G_{k}, V \in G_{t-k}$ be arbitrary. By Lemma 4 , there exists a matrix $R \in G_{t}$ such that

$$
\begin{array}{ll}
R \equiv I \quad\left(\bmod n_{r}\right), & 1 \leqq r \leqq t-1, r \neq k, \\
R \equiv\left[\begin{array}{ll}
U & 0 \\
0 & V
\end{array}\right] \quad\left(\bmod m_{k} n_{k}\right) . &
\end{array}
$$

Then $R \in G_{t}(\mathrm{~mm}) \subset H$, and hence also $W_{1}=R W R^{-1} \in H$. But we have

$$
W_{1} \equiv\left[\begin{array}{cc}
I^{(k)} & 0 \\
n_{k} V Y_{k} U^{-1} & I^{(t-k)}
\end{array}\right] \quad\left(\bmod m_{k} n_{k}\right),
$$

and

$$
W_{1} \equiv\left[\begin{array}{ll}
I^{(r)} & 0 \\
n_{r} Y_{r} & I^{(t-r)}
\end{array}\right] \quad\left(\bmod n_{r}\right)
$$

for $1 \leqq r \leqq t-1, r \neq k$. The same reasoning as in the proof of Theorem 1 then shows that all lower triangular matrices in $G_{t}(\mathfrak{n})$ lie in $H$, whence $H=G_{t}(\mathfrak{n})$ and Theorem 3 is proved.

5. We conclude with an examination of the symplectic modular group $\Gamma_{t}$ of order $t$ (see [4]). Let

$$
\Gamma_{t}(m, n)=\left\{\left[\begin{array}{ll}
A^{(t)} & B \\
C & D^{(t)}
\end{array}\right] \in \Gamma_{t}: \begin{array}{l}
B \equiv 0(\bmod m), \\
C \equiv 0(\bmod n)
\end{array}\right\},
$$

and set $\Gamma_{t}(n)=\Gamma_{t}(1, n)$. We shall prove analogues of Theorems 1 and 2 . We begin with

Lemma 6. Let $n$ be a fixed positive integer, and let

$$
M=\left[\begin{array}{ll}
A^{(t)} & B \\
C & D^{(t)}
\end{array}\right] \in \Gamma_{t} .
$$

Then there exists a symmetric $t \times t$ matrix $X$ such that $(|A+X C|, n)=1$.

Proof. As in the proof of Lemma 1, it suffices to show for each prime $p$ that there exists a symmetric matrix $X_{p}$ for which $p \nmid\left|A+X_{p} C\right|$. For $U, V$ $\in G_{t}$ we have

$$
\left[\begin{array}{ll}
U & 0 \\
0 & U^{\prime-1}
\end{array}\right] M\left[\begin{array}{ll}
V & 0 \\
0 & V^{\prime-1}
\end{array}\right]=\left[\begin{array}{ll}
A_{1}^{(t)} & B_{1} \\
C_{1} & D_{1}^{(t)}
\end{array}\right] \in \Gamma_{t},
$$

with $A_{1}=U A V, C_{1}=U^{-1} C V$. Set $Y_{p}=U X_{p} U^{\prime}$; then

$$
A_{1}+Y_{p} C_{1}=U\left(A+X_{p} C\right) V .
$$

Hence we need only find a symmetric matrix $Y_{p}$ such that $p \nmid\left|A_{1}+Y_{p} C_{1}\right|$. 
By proper choice of $U, V \in G_{t}$, we may assume that $A_{1}$ is diagonal. Let

$$
A_{1} \equiv\left[\begin{array}{ll}
E^{(k)} & 0 \\
0 & 0
\end{array}\right] \quad(\bmod p)
$$

where $E$ is diagonal and nonsingular modulo $p$. (The case where $A \equiv 0(\bmod p)$ is easily disposed of separately.) Setting

$$
C_{1}=\left[\begin{array}{ll}
C_{11}^{(k)} & C_{12} \\
C_{21} & C_{22}^{(t-k)}
\end{array}\right],
$$

the symmetry of $A_{1}^{\prime} C_{1}$ shows that $C_{12} \equiv 0(\bmod p)$. Hence

$$
\left[\begin{array}{l}
A_{1} \\
C_{1}
\end{array}\right] \equiv\left[\begin{array}{ll}
E & 0 \\
0 & 0 \\
C_{11} & 0 \\
C_{21} & C_{22}
\end{array}\right] \quad(\bmod p)
$$

whence $p \nmid\left|C_{22}\right|$. Then set

$$
Y_{p}=\left[\begin{array}{ll}
0 & 0 \\
0 & I^{(t-k)}
\end{array}\right]
$$

and obtain

$$
A_{1}+Y_{p} C_{1} \equiv\left[\begin{array}{ll}
E & 0 \\
C_{21} & C_{22}
\end{array}\right] \quad(\bmod p)
$$

which shows that $p \nmid\left|A_{1}+Y_{p} C_{1}\right|$. This completes the proof of the lemma.

Lemma 7. Let $M \in \Gamma_{t}(n)$, and let $m$ be a fixed positive integer. Then there exist symmetric integral $t \times t$ matrices $X, Y$, whose entries are determined only modulo $m$, such that

$$
W(n Y) S(X) M \in \Gamma_{t}(m n)
$$

where.

$$
W(n Y)=\left[\begin{array}{ll}
I^{(t)} & 0 \\
n Y & I^{(t)}
\end{array}\right], \quad S(X)=\left[\begin{array}{ll}
I^{(t)} & X \\
0 & I^{(t)}
\end{array}\right] .
$$

Proof. The proof follows that of Lemma 2. The only additional fact needed is that the matrix $Y$ determined by Equation (11) can be chosen to be symmetric, since the symmetry of $A_{0}^{\prime} C$ implies that of $C A_{0}^{\text {adj }}$.

We now have

Theorem 4. Let $m, n$ be positive integers, and let II be a group such that

$$
\Gamma_{t}(m n) \subset H \subset \Gamma_{t}(n) .
$$


Then there exists a divisor $d$ of $m$ such that $H=\Gamma_{t}(d n)$.

Proof. This theorem follows from Lemmas 6 and 7 in the same manner that Theorem 1 follows from Lemmas 1 and 2. We omit the details.

THEOREM 5. Let $m, n$ be positive coprime integers, and let $H$ be a group satisfying

$$
\Gamma_{t}(m, n) \subset H \subset \Gamma_{t} .
$$

Then there exist integers $m_{1}, n_{1}$ with $m_{1}\left|m, n_{1}\right| n$, and $H=\Gamma_{t}\left(m_{1}, n_{1}\right)$.

Proof. The proof of Theorem 2 carries over to this case with minor modifications. We omit the details.

\section{REFERENCES}

1. Morris Newman, Structure theorems for modular subgroups, Duke Math. J. vol. 22 (1955) pp. 25-32.

2. - An inclusion theorem for modular groups, Proc. Amer. Math. Soc. vol. 8 (1957) pp. $125-127$.

3. Irving Reiner and J. D. Swift, Congruence subgroups of matrix groups, Pacific J. Math. vol. 6 (1956) pp. 529-540.

4. L. K. Hua and Irving Reiner, On the generators of the symplectic modular group, Trans. Amer. Math. Soc. vol. 65 (1949) pp. 415-426.

National Bureau of Standards,

Washington, D. C.

UNIVERSITY OF ILLINOIS,

URBANA, ILL. 